\title{
Calendar time as an instrumental variable in assessing the risk of heart failure with antihyperglycemic drugs
}

\author{
Mugdha Gokhale ${ }^{1,2}$ (D) | John B. Buse ${ }^{3}$ | Christina DeFilippo Mack ${ }^{1,4}$ | \\ Michele Jonsson Funk ${ }^{1}$ | Jennifer Lund ${ }^{1}$ | Ross J. Simpson ${ }^{3}$ | Til Stürmer ${ }^{1}$ (D)
}

\footnotetext{
${ }^{1}$ Department of Epidemiology, Gillings School of Global Public Health, University of North Carolina at Chapel Hill, Chapell Hill, NC, USA

${ }^{2}$ Real World Evidence \& Epidemiology, GlaxoSmithKline, Collegeville, PA, USA

${ }^{3}$ Department of Medicine, University of North Carolina School of Medicine, Chapell Hill, NC, USA

${ }^{4}$ Real-World and Late Phase Research, Quintiles, Research Triangle Park, NC, USA

Correspondence

M. Gokhale, Department of Epidemiology, Gillings School of Global Public Health, University of North Carolina at Chapel Hill, 2106 McGavran-Greenberg Hall, Chapel Hill, NC, USA.

Email: mugdha.n.gokhale@gsk.com;

mgokhale@unc.edu

Funding information

National Institute on Aging, Grant/Award Number: R01 AG023178; CER Strategic Initiative of UNC's Clinical Translational Science Award, Grant/Award Number: UL1TR001111; Pharmacoepidemiology Gillings Innovation Lab (PEGIL) for the Population-Based Evaluation of Drug Benefits and Harms in Older US Adults, Grant/Award Number:

GIL200811.0010
}

\begin{abstract}
Objective: In recent years, second-line diabetes treatment with dipeptidyl peptidase-4 inhibitors (DPP-4i) increased with a corresponding decrease in thiazolidinediones (TZDs). Using hospitalization for heart failure (HF) as a positive control outcome, we explored the use of calendar time as an instrumental variable (IV) and compared this approach to an active comparator new-user study.

Methods: We identified DPP-4i or TZD initiators after a 6-month washout using Medicare claims 2006-2013. The IV was defined as a binary variable comparing initiators during October 2010 to December 2013 (postperiod) versus January 2008 to May 2010 (preperiod). We examined IV strength and estimated risk differences (RDs) for HF using Kaplan-Meier curves, which were compared with propensity score (PS)-weighted RD for DPP-4i versus TZD.
\end{abstract}

Results: The IV compared 22696 initiators (78\% DPP-4i) in the postperiod versus 20283 initiators (38\% DPP-4i) in the preperiod, resulting in 40\% compliance. The active-comparator (PS-weighted) approach compared 26198 DPP-4i and 18842 TZD initiators. Covariate balance across IV levels was slightly better than across treatments (standardized difference, $3 \%$ vs 4.5\%). The 1- and 2-year local average treatment effects of RD of HF per 100 patients in the "compliers" (95\% confidence intervals) were $-0.62(-0.99$ to -0.25$)$ and $-0.88(-1.46$ to -0.25$)$. Corresponding PS-weighted results were $-0.20(-0.33$ to -0.05$)$ and $-0.18(-0.30$ to 0.03$)$.

Conclusion: Both approaches indicated lesser risk of HF hospitalizations among DPP-4i vs TZD initiators. The magnitude of the estimated effects may differ due to differences in the target populations and assumptions. Calendar time can be leveraged as an IV when market dynamics lead to profound changes in treatments.

\section{KEYWORDS}

calendar time, instrumental variables, pharmacoepidemiology

\section{1 | INTRODUCTION}

Antihyperglycemic drugs are critical components of diabetes management, accounting for approximately $12 \%$ of diabetes costs. ${ }^{1-3}$

This work was presented at the 32nd ICPE in Dublin, Ireland, in August 2016. This is not simultaneously under consideration for publication anywhere else.
While several new antidiabetic drugs were introduced in the market in the last decade, there also emerged safety concerns about thiazolidinediones (TZDs), an established class of antihyperglycemic drugs. In 2007, a meta-analysis raised concerns over the cardiovascular safety of rosiglitazone, ${ }^{4}$ after which it was pulled from the European market, ${ }^{5}$ and in September 2010, the Food and Drug 
Administration (FDA) announced that its use would be severely restricted in the United States. ${ }^{6}$ Also in 2010, the FDA released a drug safety communication about the risk of bladder cancer with pioglitazone $^{7}$ and in 2011 approved updates to the pioglitazone product labels to include this warning. ${ }^{8}$ According to a recent analy$\mathrm{sis}^{3}{ }^{3}$ the dipeptidyl peptidase-4 (DPP-4i) class quickly gained significant market share and was the most commonly prescribed new drug class by 2012, but the use of TZDs decreased by 2012 .

In observational studies, an active comparator balances the treatment cohorts with respect to measured and unmeasured confounders, thereby minimizing the potential for confounding by indication. ${ }^{9}$ Dipeptidyl peptidase-4 and TZD are both recommended as secondline antihyperglycemic drugs, with intermediate (DPP-4i) to high (TZD) efficacy, low risk of hypoglycemia, ${ }^{10}$ and comparable costs before the generic pioglitazone was introduced in August 2012. ${ }^{11}$ The DPP-4i and TZD drugs are therefore expected to be in therapeutic equipoise in a population excluding anyone with clear contraindications. ${ }^{12}$ The increased use of DPP-4i with a simultaneous decrease in TZD use over a short period also provides an opportunity to use calendar time as an instrumental variable (IV). ${ }^{13}$ Instrumental variable methods serve as a natural experiment to compare drugs in settings where substantial unmeasured confounding is expected. ${ }^{14-16}$ Common IV in pharmacoepidemiology includes provider preference, calendar time, distance to care, and geographic variation. ${ }^{17}$ Calendar time IV arises from secular trends in medication use. Such trends can be a result of effectiveness or safety releases, changes in physician preferences, formularies, or the introduction of new agents to the market. Calendar time IV has been previously used in several settings including studies of antidiabetic therapies on body mass index, ${ }^{18}$ colon cancer treatments on survival, ${ }^{13}$ antivirals in HIV patients, ${ }^{19}$ hormone replacement therapy, and myocardial infarction. ${ }^{20}$

Our study setting is unique as there exist both an active comparator and also a potential calendar time IV arising from drug safety concerns. Using hospitalization for heart failure (HF) as a positive control outcome (increased HF risk with TZDs, not with DPP-4i drugs sitagliptin and alogliptin), ${ }^{21-23}$ we first explored the use of calendar time as an IV. Next we compared this approach with an active comparator new-user study examining DPP-4i versus TZD in a sample of Medicare beneficiaries without prior HF.

\section{2 | METHODS}

We used a 20\% random sample of the 2007-2013 Medicare claims data for this study. These data contain information about demographics, enrollment, inpatient, outpatient diagnoses, procedures, and prescription drugs for each enrollee. ${ }^{24,25}$

From a population of Medicare beneficiaries aged $>65$ years, we identified new users of DPP-4i or TZD without the use of either drugs in the prior 6 months. Index date was defined as the date of the first prescription. Since TZDs are contraindicated in patients with existing $\mathrm{HF},{ }^{26}$ we excluded patients with diagnoses of HF and related conditions (cardiomyopathy, arrhythmias, chronic kidney disease, edema, and loop diuretic use). We required the patients to have at least 6 months of continuous part D enrollment and at least 12 months

\section{KEY POINTS}

- In recent years, second-line treatment of patients with type 2 diabetes with dipeptidyl peptidase-4 inhibitors (DPP-4i) increased with a corresponding decrease in thiazolidinediones (TZDs). This crossover over a short period may provide an opportunity to use calendar time as an instrumental variable (IV). Using hospitalization for heart failure (HF) as a positive control outcome, we explored the use of calendar time as an IV and compared this approach to an active comparator new-user study comparing DPP versus TZD.

- The binary IV defined based on drug initiation patterns and the timing of safety signals compared initiators during October 2010 to December 2013 ("postperiod") versus January 2008 to May 2010 ("preperiod"), resulting in $40 \%$ compliance, which implies this is a strong IV. Using Kaplan-Meier methods, we estimated the 1- and 2-year risk differences for HF hospitalization using 2 approaches: (1) IV approach comparing postperiod versus preperiod and (2) active comparator approach comparing DPP-4i versus TZD initiators using propensity score weighting.

- Both approaches including many sensitivity analyses indicated lesser risk of HF hospitalizations among DPP-4i vs TZD initiators. The magnitude of the estimated effects may differ due to differences in the assumptions and target populations.

- Our results demonstrate that calendar time can be leveraged as an IV when market dynamics lead to profound changes in treatments over short periods.

parts $A$ and $B$ enrollment preindex. To ensure that patients were actually started on the drugs, we restricted our cohorts to patients with a second prescription for the same drug class dispensed within 6 months after the index date.

\section{1 | Instrumental variable}

An IV is an observed variable associated with the variation in exposure similar to randomized assignment. ${ }^{16}$ To be valid, an IV must be (1) associated with the treatment, (2) unrelated to patient characteristics, and (3) related to the outcome only through its association with the treatment. Additionally, to estimate the average treatment effect in the "compliers," the instrument should affect treatment deterministically in one direction (monotonicity assumption). ${ }^{16}$

We defined the IV as a binary variable ("postperiod" versus "preperiod") anchored around the time of the crossover of the drug initiation curves (June to September 2010), which was also the time when the FDA issued safety communications about TZDs. In our study, compliers are patients initiating TZD before the crossover and DPP-4i after the crossover. We identified an "optimal" IV by evaluating the percentage of compliers (the strength of the instrument's 
effect on the received treatment). ${ }^{13}$ We examined the instrument in relation to IV assumptions using the proportion of compliers, partial $F$ tests, measured covariates, and expert knowledge. In IV analyses, the approach of examining covariate balance by only looking at the standardized absolute mean differences (SAMDs) ignores the scaling factor and can be potentially misleading while evaluating confounding bias. Therefore, we evaluated covariate balance with the "actual treatment initiated" approach and IV approach by using both the SAMDs and bias components taking into account scaling of the IV as proposed by Jackson et al. ${ }^{27}$ The binary measure of calendar time was decided before examining the effect estimates.

\section{2 | Outcome}

Heart failure hospitalization was defined as an inpatient claim with International Classification of Diseases, Ninth Revision, code 428.xx in the primary position. ${ }^{28}$ Thiazolidinediones are known to increase HF risk, ${ }^{21}$ and while saxagliptin is suspected to be associated with an increased HF risk, other DPP-4i drugs (constituting $>80 \%$ of our cohort, Table S1) are not. ${ }^{22,23,29}$ Heart failure hospitalization is therefore a positive control outcome in our study enabling the comparison of the analysis "comparing the actual treatments initiated" versus the "IV analysis comparing levels of the calendar time instrument." Using an intent-to-treat (ITT) approach, patients were followed from the second prescription date until the earliest of HF hospitalization, death, end of enrollment, or 2 years after the index date.

\section{3 | Confounding control and analysis}

We used weighted Kaplan-Meier methods to estimate the 1- and 2year risk differences (RDs) for HF hospitalization comparing (a) actual treatment received (DPP-4i vs TZD) and (b) IV approach (postperiod versus preperiod). Under the "treatment received" approach, we first estimated the propensity score (PS) for DPP-4i versus TZD ${ }^{30}$ using logistic regression with baseline variables measured preinitiation. The year of initiation was not included in PS estimation because it was a suspected instrument strongly associated with the exposure but not with the outcome, and conditioning on such a variable could bias the results and increase variance. ${ }^{31} \mathrm{We}$ then assigned inverse probability of treatment weights (IPTW) of 1/PS to DPP-4i and 1/(1 - PS) to TZD and stabilized both groups by marginal prevalence of the treatment actually received. ${ }^{32}$ Using IPTW-weighted Kaplan-Meier curves, we estimated the RDs for HF hospitalization with treatment received as the strata variable and with a robust variance estimator. ${ }^{33}$

The effect of the IV levels on HF hospitalization was observed by the IV estimates of 1- and 2-year RDs scaled by IV strength to estimate the local average treatment effect among compliers. In a secondary analysis, we generated covariate-adjusted IV estimates of the RD using weighted Kaplan-Meier methods analogous to the IPTW-weighted method and scaled this by IV strength.

These methods are appreciably different-IV estimates apply to the marginal patients while IPTW PS methods apply to the whole study population. Propensity score models used the full study population, and we performed sensitivity analyses in the reduced IV cohort to evaluate selection differences that may have been introduced based on IV exclusions. ${ }^{13}$ Next, we performed a sensitivity analysis restricting the study cohort to patients who initiated treatment between July 2007 and December 2011, with follow-up through December 2013. This approach excluded anyone who initiated therapy during or after January 2012, who would not have the potential for a 2-year follow-up before the end of the study). We also performed additional analyses with different definitions of the instrument to assess the effects of potential time trends in the outcome and effects of scaling by compliance percentage (Figures S2 and S3).

\section{3 | RESULTS}

The preferred treatments changed dramatically over the study period (Figure 1). The optimal binary IV compared the postperiod (October 2010 to December 2013, N = 22 696) with the preperiod (January 2008 to May 2010, N = 20 283). This definition excludes patients during June 2010 to September 2010 where the curves intersect. With this IV definition, the DPP-4i treatment rates were $78 \%$ and $38 \%$ in the postperiod and preperiod, respectively, and the strength

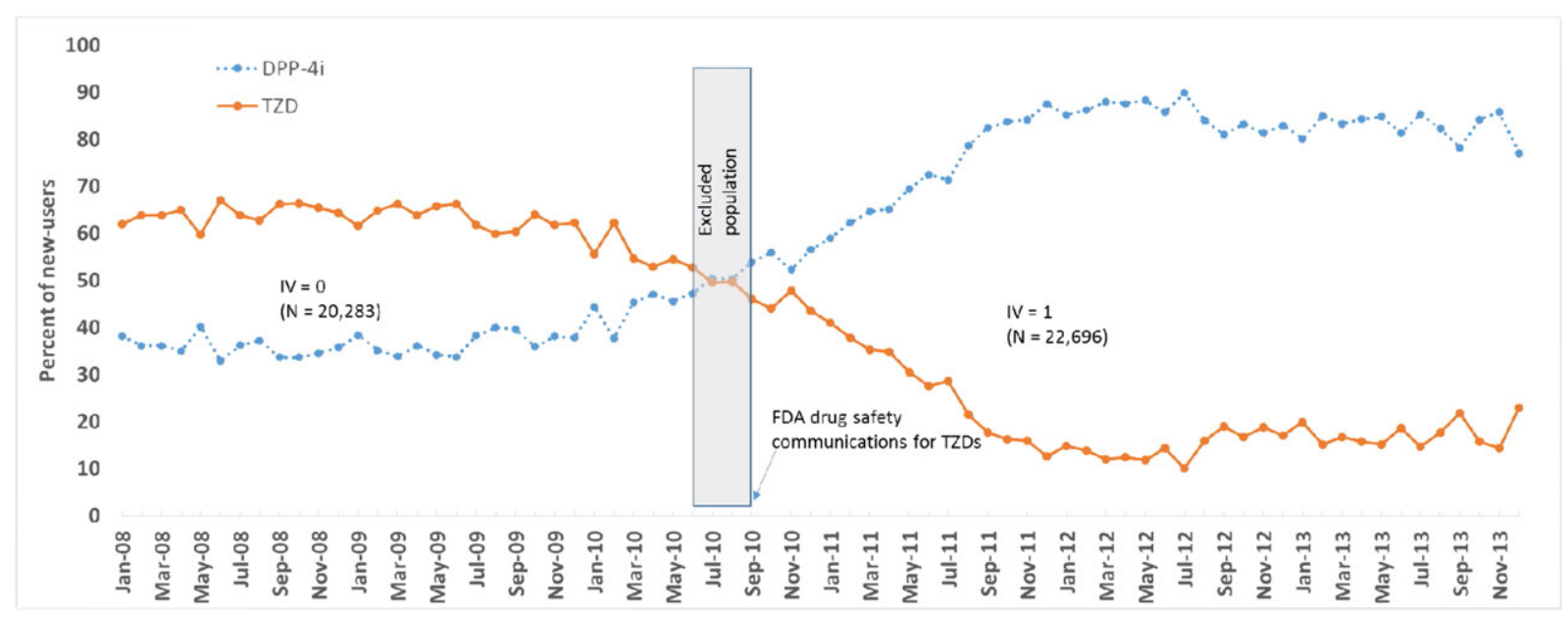

FIGURE 1 Initiation of dipeptidyl peptidase 4 inhibitors (DPP-4i) versus thiazolidinediones (TZD) across calendar time and the resultant definition of the instrumental variable (IV): Medicare claims data 2007-2013 [Colour figure can be viewed at wileyonlinelibrary.com] 
of the IV (\% compliers) was $40 \%$. The partial $F$ statistic assessing the association between the treatment and calendar time was 4596.7.

While the covariates were well balanced across DPP-4i versus TZD, the balance was slightly better across the levels of the IV as illustrated by the average SAMD of $4.5 \%$ vs $3.0 \%$, respectively (Table 1 ). This indicates that the IV is independent of the measured risk factors of the outcome and potentially also unmeasured covariates. We also calculated the bias components to compare the covariate balance for the 2 analytic approaches (Table 1, last 2 columns). The bias component for the IV approach was relatively higher than the bias component with the "actual treatment initiated" approach for key variables like diabetic neuropathy, retinopathy, and baseline use of metformin and sulfonylureas, but the magnitude of the SAMD with both approaches was $<10 \%$.

In the full population comparing DPP-4i versus TZD, the crude incidence rate per 100000 was 563 in DPP-4i and 676 in TZD initiators (Table 2). The 1- and 2-year risks for HF hospitalization were $0.5 \%$ and $1.2 \%$ for DPP-4i and $0.7 \%$ and $1.3 \%$ for TZD, respectively (Figure S1). In the reduced IV population, crude incidence rate per 100000 person-years was 509 in the postperiod and 695 in the preperiod. The 1- and 2-year risks for HF hospitalization were $0.4 \%$ and $1.1 \%$ in the postperiod and $0.7 \%$ and $1.4 \%$ in the preperiod, respectively.

The crude RD was similar to the IPTW-weighted RD (Figure 2) obtained by comparing DPP-4i versus TZD, expected because of the balance of baseline covariates achieved by the use of an active comparator. The IV estimates of the RDs scaled by IV strength were consistent overall with the IPTW-weighted RDs indicating a protective effect with the DPP-4i but relatively less precise and farther from the null. The 2-year RD per 100 patients obtained using the IV analysis scaled by IV strength was $-0.62(-0.99$ to -0.25$)$ indicating that of the 1000 compliant patients treated with DPP-4i, 6 fewer patients would be expected to have an HF hospitalization during the first year relative to those with TZD. Covariate-adjusted IV estimates of the RD were similar (Figure 2, Table S2).

Sensitivity analyses in a population restricted to patients initiating on or before December 2011, analyses using IPTW weighting in the reduced IV population (Table S3), and analyses using different definitions of the IV to assess the effects of scaling and potential outcome time trends yielded similar results (Figures S2 and S3).

\section{4 | DISCUSSION}

This study compared the performance of 2 methods to estimate the effect of DPP-4i versus TZD initiation on the risk of HF hospitalization, a positive control outcome. The first method used an active comparator design using IPTW weighting, while the second method compared posttime versus pretime using calendar time as an IV and found lesser risk of HF hospitalization with DPP-4i.

The absolute 1-year risks of HF hospitalization in the sitagliptin and pioglitazone arms (together accounting for approximately $85 \%$ of our population; Table S1) were approximately $1 \%$ and $2 \%$, respectively, in previous randomized trials. ${ }^{21,23}$ These trials found no increased risk with sitagliptin and $0.5 \%$ increased risk with pioglitazone relative to placebo at 1 year; however, there are no data directly comparing DPP-4i with TZD. In our study, the claims-based HF definition had a high specificity but a low sensitivity, ${ }^{28}$ which led to an underestimation of absolute HF risks and therefore also RDs for both the IPTW and IV methods. While we could have chosen to estimate relative risks, we chose to estimate RD because we were interested in an absolute measure of effect, which is more interpretable and relevant in our setting. Moreover, different IV methods used to derive relative measures require different additional assumptions like a special type of noncompliance (noncompliers are only those who do not take the treatment when assigned to the treatment) or about the homogeneity of risks within the levels of the treatment. ${ }^{16,34}$

While the RDs from both approaches generally agreed, the IV estimates were less precise, and the magnitudes of the RDs differed because the 2 methods apply to different populations and are based on different assumptions. The IPTW-weighted RD is the average treatment effect in the entire population, while the scaled RD from the IV analysis is the average treatment effect in the $40 \%$ compliers from the reduced IV population. Scaling the RD involves dividing the RD by the IV strength of $40 \%$. The weaker the instrument, the larger the scaled estimate gets relative to the unscaled RD, potentially magnifying any biases present in the unscaled RD. ${ }^{16}$ In a sensitivity analysis (Figure S2), we defined the instrument by a cut-point (before and after July 2010), which made this a weaker instrument (IV strength, $25 \%$ ), and the scaled 1-year RD was -0.73 (-1.74 to 0.27$)$, which has a larger magnitude than the main analysis, but in the same direction. In another analysis (Figure S3), we compared the periods with maximum separation (December 2011 to December 2012 versus June 2008 to June 2009). This definition led to a stronger IV (strength, $50 \%$ ), and as expected, the scaled 1-year RD was closer to the unscaled estimate $(-0.45 ; \mathrm{Cl},-0.92$ to 0.05$)$. Thus, scaling could explain some of the differences in magnitude of the IV estimates of RDs compared with the IPTW-weighed RDs seen in the main analysis. We compared the characteristics of the compliers with that of the full population and found that the populations were generally similar with minor differences insufficient to explain our results (Table S4).

It is also important to critically assess IV assumptions. While we empirically observed that calendar time strongly affected the treatment initiation, the assumption of calendar time being unrelated to the outcome through other mechanisms could be potentially violated due to time trends in the outcome. However, no strong trends in HF hospitalizations were empirically observed in the population excluded from our study due to previous HF or other criteria (between $3.1 \%$ to $4.6 \%$ of the initiators were excluded each year during the study period due to a previous HF hospitalization). The literature reports reduction in the rates of HF hospitalization among Medicare beneficiaries due to several reasons including decreased smoking and increasing outpatient management. ${ }^{35}$ The Hospital Readmissions Reduction Program started in late 2012 requires Centers for Medicare \& Medicaid Services (CMS) to penalize hospitals with excess readmissions for certain conditions including HF, which may somewhat explain the decreasing trend in HF hospitalizations, but since this only affects a small proportion of our study period, this is not expected to meaningfully affect our results. ${ }^{36}$

Assuming a decreasing trend in the rates of HF hospitalization during the study period, the estimates of RD are expected to be biased 


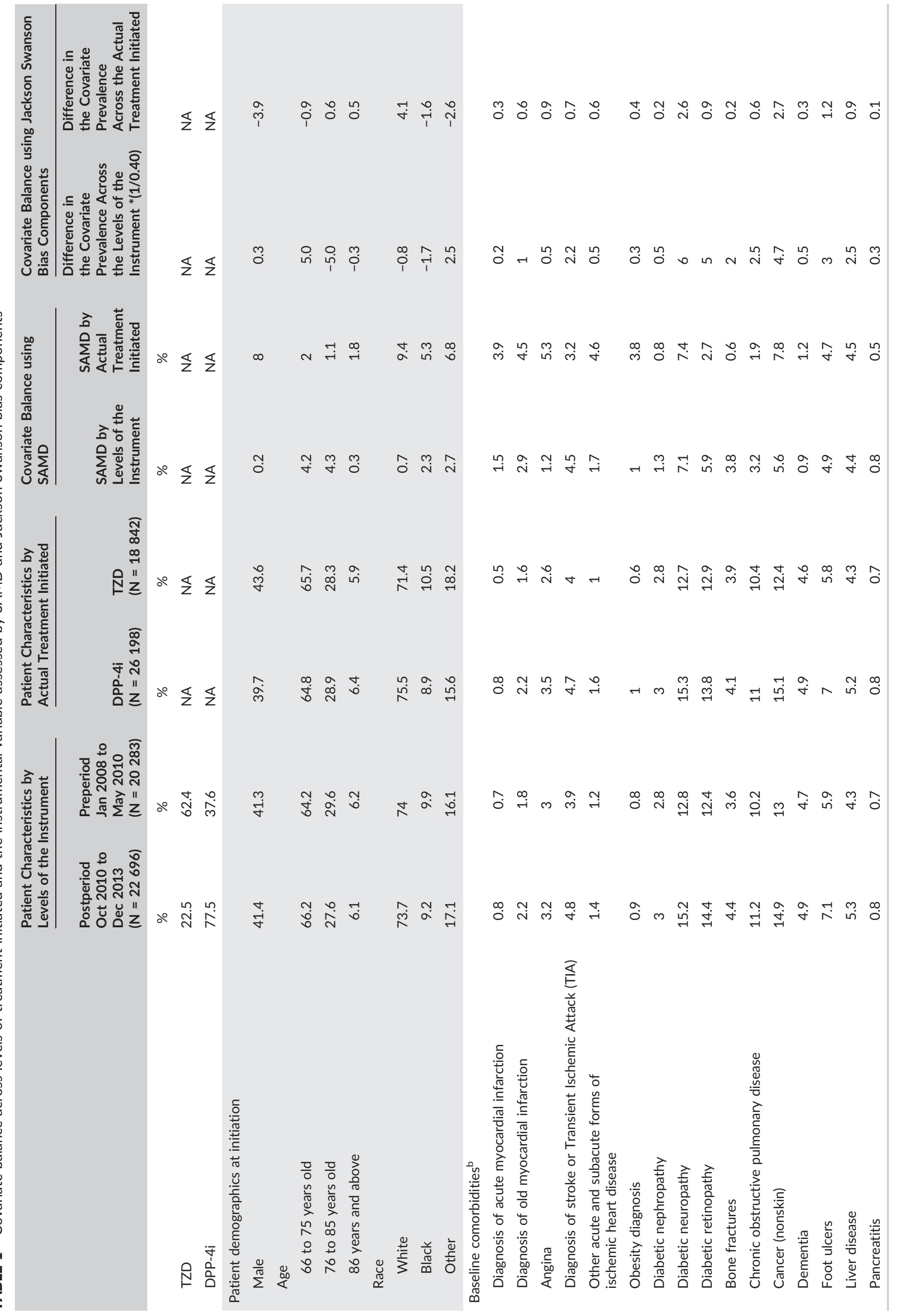




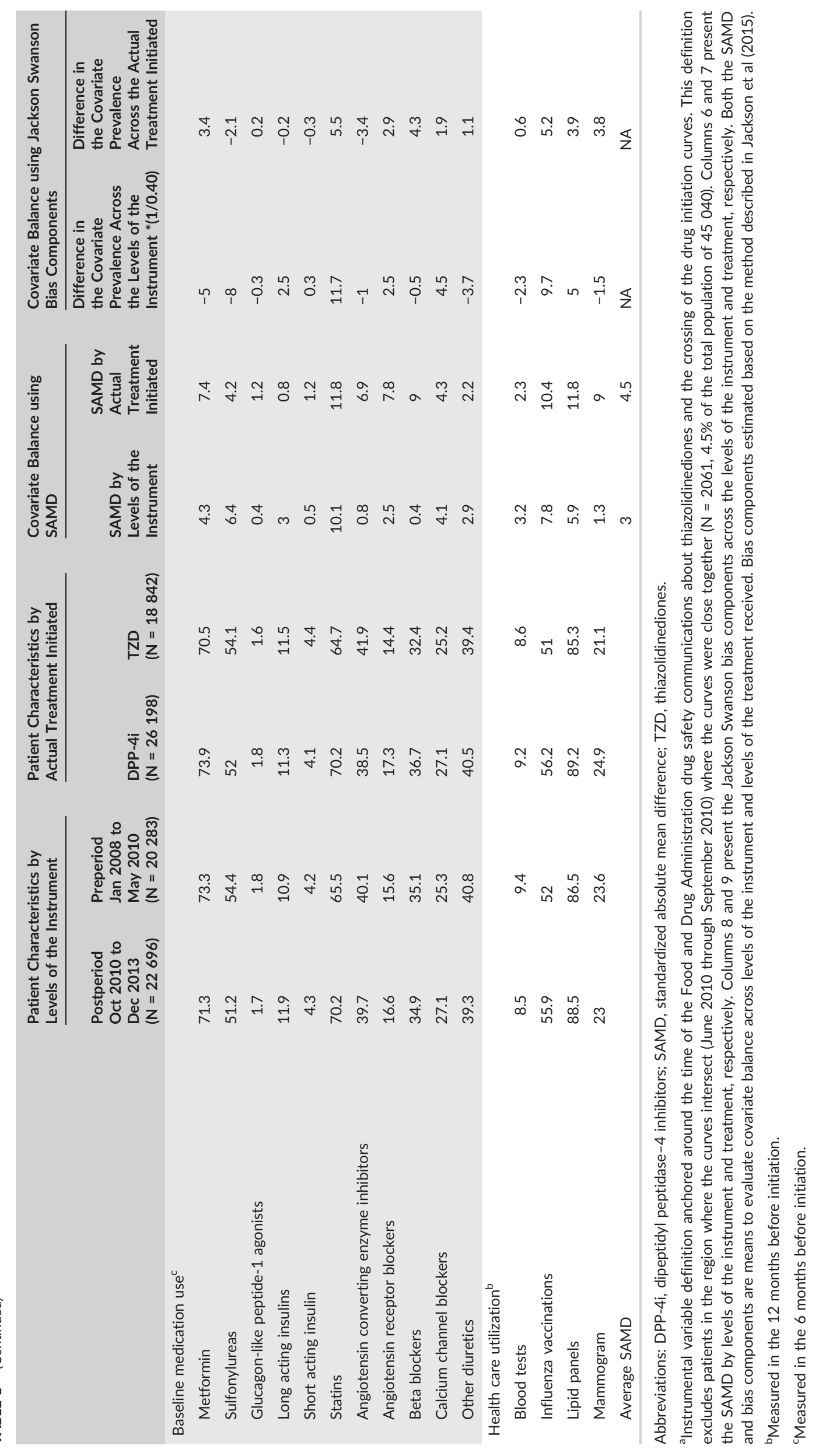


TABLE 2 Number of new users, heart failure hospitalizations, time on treatment, total person time, and incidence for comparisons of levels of the instrument and treatment initiated: Medicare claims data 2007-2013

\begin{tabular}{|lllllll} 
Comparison & Level & $\begin{array}{l}\text { Number of } \\
\text { New Users }\end{array}$ & Events & $\begin{array}{l}\text { Duration of Follow-up in Years } \\
\text { Interquartile Range (Median) }\end{array}$ & $\begin{array}{l}\text { Total Person- } \\
\text { years }^{\text {b }}\end{array}$ & $\begin{array}{l}\text { Incidence (per } 100 \text { 000 } \\
\text { Person- years) }\end{array}$ \\
\hline Post vs Pre ${ }^{\mathrm{a}}$ & Post & 22696 & 146 & $1.36(0.64-2.00)$ & 28661 & 509.40 \\
& Pre & 20283 & 255 & $2.00(2.00-2.00)$ & 36682 & 695.16 \\
DPP-4i vs TZD & DPP-4i & 26198 & 210 & $2.00(1.79-2.00)$ & 37252 & 563.73 \\
& TZD & 18841 & 215 & $2.00(1.68-2.00)$ & 31791 & 676.29 \\
\hline
\end{tabular}

Abbreviations: DPP-4i, dipeptidyl peptidase-4 inhibitors; TZD, thiazolidinedione.

aPost, October 2010 to December 2013; pre, January 2008 to May 2010.

${ }^{b}$ Under the first treatment carried forward/intent-to-treat analysis, patients were followed up from the second prescription of the initiated drug to earliest of the outcome occurrence, death, or 2 years after initiation without accounting for treatment changes during follow-up.

FIGURE 2 Comparison of risk differences (RDs) for heart failure hospitalization: Medicare claims data 2007-2013. Estimates of risk difference are based on risks per 100 patients estimated from Kaplan-Meier survival curves. The instrumental variable (IV) estimator is scaled by a compliance percentage of $40 \%$. Adjusted estimates account for the variables presented in Table 1. IPTW, inverse probability of treatment weight; PS, propensity score [Colour figure can be viewed at wileyonlinelibrary.com]

\section{(A) - 1-year risk differences}

0.1

บ -0.1

ํํ -0.3

단 -0.5

\& -0.7

产 -0.9

过 -1.1

产 -1.3

$-1.3$

$-1.5$

Unadjusted IV estimator IV estimator Adjusted IV

unscaled

scaled by

estimator

scaled by

compliance

PS IPTW

weighted

(B) - 2-year risk differences

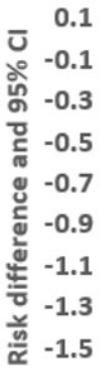

I

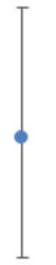

Unadjusted IV estimator IV estimator Adjusted IV unscaled

scaled by compliance

estimator

scaled by

compliance

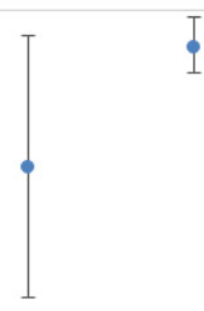

PS IPTW weighted 
diabetic neuropathy, retinopathy, baseline use of metformin, and sulfonylureas, indicating that not adjusting for these variables in the IV analyses would result in more bias than not adjusting them in the conventional PS-weighted active comparator analysis. However, the bias component method only gives an idea about the relative bias but not absolute bias. ${ }^{27}$ In this study, the magnitude of the SAMD for both approaches was $<10 \%$ for these variables, and our adjusted and unadjusted IV estimates are only slightly different indicating no significant concern for confounding by these variables in our main analysis. The assumption of monotonicity also seems to be reasonable in this study as it is unlikely that a patient would initiate DPP-4i in the preperiod while an identical patient would initiate TZD in the postperiod in spite of the warnings about TZD. Any impact of the generic pioglitazone in August 2012 on this study would be in the last year only and therefore unlikely to be a serious concern. There were no events that would preclude DPP-4i initiator in the preperiod from receiving DPP-4i in the postperiod, especially given the reports suggesting a lack of serious adverse events with these agents in the past few years. ${ }^{37,38}$

Some additional caveats should be considered. Our ITT analysis approach could lead to exposure misclassification as it does not take into account treatment changes. However, the IV estimator is a measure of the association between the treatment assignment (i.e., treatment intent) and the outcome. Therefore, using a conceptually uniform ITT approach for the IPTW-weighted analysis comparing DPP-4i versus TZD initiation would facilitate direct comparison of the RDs obtained by the 2 methods. Finally, it could be argued that confounding exists in the DPP-4i versus TZD comparison by unmeasured factors like body mass index or smoking. However, body mass index is not likely to affect the choice of initiation of DPP-4i versus TZD in these patients, and chronic obstructive pulmonary disease was available as a proxy for smoking, which reduces the concern of confounding by these variables. ${ }^{39}$

A key strength of our study was the identification of a calendar time IV that strongly affected the exposure, driven by the dynamics of the antihyperglycemic drug market. Time trends in the exposure also offer other study design options like secular trend analyses. Compared with a secular trend analysis that is more useful in forecasting future outcomes and generating hypotheses, using calendar time as a binary IV in this study enabled clear visualization of the measured patient characteristics by levels of the IV and enabled conditioning on any remaining imbalances while at the same time also checking if there is a trend in the outcome. These features make the IV method more intuitive while drawing conclusions about comparative performance of the treatments of interest.

It is often conservatively suggested to avoid IV methods when the assumptions are likely to be violated because relatively minor violations of the assumptions may lead to large biases. ${ }^{14}$ This can be particularly problematic when there is no valid comparison analytic method. In our setting, the use of a positive control outcome and observing consistent results with both IV and IPTW methods imply that both may be equally valid and supplement each other at the very least. Our results indicate that calendar time can be leveraged as a strong IV in settings where market dynamics lead to profound changes in preferred treatments.

\section{FUNDING}

This work was supported by the following: The Medicare database infrastructure used for this was funded by the Pharmacoepidemiology Gillings Innovation Lab (PEGIL) for the Population-Based Evaluation of Drug Benefits and Harms in Older US Adults (GIL200811.0010), the Center for Pharmacoepidemiology, Department of Epidemiology, the CER Strategic Initiative of UNC's Clinical Translational Science Award (UL1TR001111), the Cecil G. Sheps Center for Health Services Research, and the School of Medicine. The development of the stateof-the art nonexperimental methodology was supported by an ongoing grant from the National Institute on Aging (R01 AG023178).

\section{ETHICS STATEMENT}

The authors state that no ethical approval was needed.

\section{CONFLICT OF INTEREST}

Dr Gokhale was a doctoral student at University of North Carolina during the conduct of this study. She is now a full-time employee of GlaxoSmithKline.

Dr Buse reports grants, nonfinancial support, and other from Eli Lilly, Bristol-Myers Squibb, GI Dynamics, Merck, AstraZeneca, Sanofi, Lexicon, Novo Nordisk, Orexigen, and Takeda, nonfinancial support and other from Elcylex, Metavention, vTv Pharma, Dance Biopharm, Quest, and Adocia, grants, personal fees, nonfinancial support, and other from PhaseBio, and grants from Medtronic Minimed, Tolerex, Osiris, Halozyme, Johnson and Johnson, Andromeda, BoehringerIngelheim, GlaxoSmithKline, Astellas, MacroGenics, Intarcia Therapeutics, and Scion NeuroStim, outside the submitted work, and he has been a member of a variety of nonprofit boards: American Diabetes Association, DiabetesSisters, Taking Control of Your Diabetes, AstraZeneca Healthcare Foundation, Bristol-Myers Squib Together on Diabetes Foundation, and the National Diabetes Education Program.

Dr Stürmer receives investigator-initiated research funding and support as Principal Investigator (R01 AG023178) from the National Institute on Aging (NIA) and as Co-Investigator (R01 CA174453, R01 HL118255, R21-HD080214), National Institutes of Health (NIH). He also receives salary support as Director of the Comparative Effectiveness Research (CER) Strategic Initiative, NC TraCS Institute, UNC Clinical and Translational Science Award (UL1TR001111), and as Director of the Center for Pharmacoepidemiology (current members: GlaxoSmithKline, UCB BioSciences, and Merck) and research support from pharmaceutical companies (Amgen, AstraZeneca) to the Department of Epidemiology, University of North Carolina at Chapel Hill. Dr Stürmer does not accept personal compensation of any kind from any pharmaceutical company. He owns stock in Novartis, Roche, BASF, AstraZeneca, Johnson and Johnson, and Novo Nordisk.

Dr Lund receives research support from the UNC Oncology Clinical Translational Research Training Program (K12 CA120780), as well as through a Research Starter Award from the PhRMA Foundation to the UNC Department of Epidemiology.

Dr Jonsson Funk receives investigator-initiated research funding and support as Principal Investigator from the National Institutes of 
Health (NIH), National Heart Lung and Blood Institute (NHLBI, R01 HL118255), and as a Co-Investigator from the NIH National Institute on Aging (NIA, R01 AG023178), the NIH National Center for Advancing Translational Sciences (NCATS, 1UL1TR001111), AstraZeneca, and the Patient-Centered Outcomes Research Institute (PCORI, 1IP2PI000075). She is a member of the Scientific Steering Committee (SSC) for a postapproval safety study of an unrelated drug class funded by GSK. All compensation for services provided on the SSC is invoiced by and paid to UNC Chapel Hill.

Dr Jonsson Funk does not accept personal compensation of any kind from any pharmaceutical company, though she receives salary support from the Center for Pharmacoepidemiology in the Department of Epidemiology, Gillings School of Global Public Health (current members: GlaxoSmithKline, UCB BioSciences, and Merck).

Dr Mack is a full-time employee of Real-World and Late Phase Research, Quintiles, USA, and provides scientific consultation on studies for various pharmaceutical companies.

Dr Simpson has no conflicts of interest to report.

\section{AUTHOR CONTRIBUTIONS}

All authors participated in study conception and design and addressing reviewer comments. M.G., J.B., and T.S. participated in the acquisition of the data. M.G. did the analysis, and all authors contributed to the interpretation of the data. M.G. wrote the first draft of the manuscript. All authors reviewed and provided comments on the manuscript. M.G. is the guarantor of this work; M.G. and T.S. had full access to all the data in the study and take responsibility for the integrity of the data and the accuracy of the data analysis.

\section{ORCID}

Mugdha Gokhale (D) http://orcid.org/0000-0003-0223-1016

Til Stürmer (D) http://orcid.org/0000-0002-9204-7177

\section{REFERENCES}

1. National Diabetes Statistics Report, 2014. http://www.cdc.gov/diabetes/pdfs/data/2014-report-estimates-of-diabetes-and-its-burden-inthe-united-states.pdf. Accessed March 12, 2016.

2. American Diabetes Association. Economic costs of diabetes in the U.S. in 2012. Diabetes Care. 2013;36(4):1033-1046. https://doi.org/ $10.2337 / \mathrm{dc} 12-2625$ [doi]

3. Hampp C, Borders-Hemphill V, Moeny DG, Wysowski DK. Use of antidiabetic drugs in the U.S., 2003-2012. Diabetes Care. 2014;37(5): 1367-1374. https://doi.org/10.2337/dc13-2289 [doi]

4. Nissen SE, Wolski K. Effect of rosiglitazone on the risk of myocardial infarction and death from cardiovascular causes. N Engl J Med. 2007;356(24):2457-2471. https://doi.org/10.1056/NEJMoa072761

5. European Medicines Agency. European Medicines Agency recommends suspension of Avandia, Avandamet and Avaglim. European Medicines Agency; 2010. http://www.ema.europa.eu/ema/index.jsp? curl=pages/news_and_events/news/2010/09/news_detail_001119. jsp\&mid=WCOb01ac058004d5c1. Accessed March 12, 2016.

6. U.S. Food and Drug Administration. FDA significantly restricts access to the diabetes drug Avandia. http://www.fda.gov/Drugs/DrugSafety/ PostmarketDrugSafetylnformationforPatientsandProviders/ ucm226956.htm. Accessed March 12, 2016.

7. U.S. Food and Drug Administration. FDA drug safety communication: ongoing safety review of Actos (pioglitazone) and potential increased risk of bladder cancer after two years exposure. http://www.fda.gov/ Drugs/DrugSafety/ucm226214.htm. Accessed March 12, 2016.

8. U.S. Food and Drug Administration. FDA drug safety communication: updated drug labels for pioglitazone-containing medicines. http:// www.fda.gov/Drugs/DrugSafety/ucm266555.htm. Accessed March 12, 2016.

9. Lund JL, Richardson DB, Stürmer T. The active comparator, new user study design in pharmacoepidemiology: historical foundations and contemporary application. Curr Epidemiol Rep. 2015;2(4):221-228.

10. Inzucchi SE, Bergenstal RM, Buse JB, et al. Management of hyperglycemia in type 2 diabetes, 2015: a patient-centered approach: update to a position statement of the American Diabetes Association and the European Association for the Study of Diabetes. Diabetes Care. 2015;38(1):140-149. https://doi.org/10.2337/dc14-2441 [doi]

11. U.S. Food and Drug Administration. FDA approves first generic Actos to treat type 2 diabetes. http://www.fda.gov/NewsEvents/Newsroom/PressAnnouncements/ucm315951.htm. Accessed March 12, 2016.

12. Gokhale M, Buse JB, Jonsson $M$, et al. No increased risk of cardiovascular events in older adults initiating dipeptidyl peptidase 4 inhibitors versus therapeutic alternatives. Diabetes Obes Metab. 2017; 19(7):970-978

13. De Filippo Mack C, Brookhart MA, Glynn RJ, et al. Comparative effectiveness of oxaliplatin versus 5-flourouricil in older adults. Epidemiology. 2015;26(5):690-699.

14. Swanson SA, Hernan MA. Commentary: how to report instrumental variable analyses (suggestions welcome). Epidemiology. 2013;24(3):370-374. https://doi.org/10.1097/EDE.0b013e31828 d0590 [doi].

15. Brookhart MA, Wang PS, Solomon DH, Schneeweiss S. Evaluating short-term drug effects using a physician-specific prescribing preference as an instrumental variable. Epidemiology. 2006;17(3):268-275. https://doi.org/10.1097/01.ede.0000193606.58671.c5 [doi].

16. Brookhart MA, Rassen JA, Schneeweiss S. Instrumental variable methods in comparative safety and effectiveness research. Pharmacoepidemiol Drug Saf. 2010;19(6):537-554.

17. Swanson SA. Instrumental variable analyses in pharmacoepidemiology: what target trials do we emulate? Curr Epidemiol Rep. 2017;4(4):281-287.

18. Ertefaie A, Small DS, Flory JH, Hennessy S. A tutorial on the use of instrumental variables in pharmacoepidemiology. Pharmacoepidemiol Drug Saf. 2017;26(4):357-367.

19. Cain LE, Cole SR, Greenland S, et al. Effect of highly active anti-retroviral therapy on incident AIDS using calendar period as an instrumental variable. Am J Epidemiol. 2009;169(9):1124-1132.

20. Shetty KD, Vogt WB, Bhattacharya J. Hormone replacement therapy and cardiovascular health in the United States. Med Care. 2009; 47(5):600-606.

21. Erdmann E, Charbonnel B, Wilcox RG, et al. Pioglitazone use and heart failure in patients with type 2 diabetes and preexisting cardiovascular disease: data from the PROactive study (PROactive 08). Diabetes Care. 2007;30(11):2773-2778. doi: dc07-0717 [pii]

22. White WB, Cannon CP, Heller SR, et al. Alogliptin after acute coronary syndrome in patients with type 2 diabetes. N Engl J Med. 2013;369(14):1327-1335. https://doi.org/10.1056/NEJMoa1305889; 10.1056/NEJMoa1305889.

23. Green JB, Bethel MA, Armstrong PW, et al. Effect of sitagliptin on cardiovascular outcomes in type 2 diabetes. N Engl J Med. 2015; 373(3):232-242.

24. Medicare Program-general information. http://www.cms.gov/ Medicare/Medicare-General-Information/MedicareGenInfo/index. html. Accessed April, 2014

25. Brief Summaries of Medicare \& Medicaid. 2011. http://www.cms.gov/ Research-Statistics-Data-and-Systems/Statistics-Trends-and-Reports/ MedicareProgramRatesStats/downloads/MedicareMedicaidSummaries 2011.pdf. Accessed April, 2014. 
26. Information for Healthcare Professionals: pioglitazone $\mathrm{HCl}$ (marketed as Actos, Actoplus Met, and Duetact). http://www.fda.gov/Drugs/ DrugSafety/PostmarketDrugSafetyInformationforPatientsandProviders/ ucm124178.htm. Accessed February 3, 2016.

27. Jackson JW, Swanson SA. Toward a clearer portrayal of confounding bias in instrumental variable applications. Epidemiology. 2015;26(4):498-504. https://doi.org/10.1097/EDE.000000000000 0287 [doi].

28. Kucharska-Newton AM, Heiss $\mathrm{G}, \mathrm{Ni} \mathrm{H}$, et al. Identification of heart failure events in Medicare claims: the Atherosclerosis Risk in Communities (ARIC) study. J Card Fail. 2016;22(1):48-55.

29. Scirica BM, Bhatt DL, Braunwald E, et al. Saxagliptin and cardiovascular outcomes in patients with type 2 diabetes mellitus. N Engl J Med. 2013;369(14):1317-1326. https://doi.org/10.1056/NEJMoa1307684; 10.1056/NEJMoa1307684.

30. Rosenbaum PR, Rubin DB. The central role of the propensity score in observational studies for causal effects. Biometrika. 1983;70(1):41-55.

31. Myers JA, Rassen JA, Gagne JJ, et al. Effects of adjusting for instrumental variables on bias and precision of effect estimates. Am J Epidemiol. 2011;174(11):1213-1222. https://doi.org/10.1093/aje/ kwr364 [doi].

32. Stürmer T, Rothman KJ, Glynn RJ. Insights into different results from different causal contrasts in the presence of effect-measure modification. Pharmacoepidemiol Drug Saf. 2006;15(10):698-709.

33. Joffe MM, ten Have Thomas R, Feldman HI, Kimmel SE. Model selection, confounder control, and marginal structural models: review and new applications. Am Stat. 2004;58(4):272-279.

34. Greenland S. An introduction to instrumental variables for epidemiologists. Int J Epidemiol. 2000;29(4):722-729.

35. Krumholz HM, Normand SL, Wang Y. Trends in hospitalizations and outcomes for acute cardiovascular disease and stroke, 1999-2011.
Circulation. 2014;130(12):966-975.

https://doi.org/10.1161/ CIRCULATIONAHA.113.007787 [doi]

36. Centers for Medicare and Medicaid Services. Readmissions Reduction Program (HRRP). https://www.cms.gov/medicare/medicare-fee-forservice-payment/acuteinpatientpps/readmissions-reduction-program. html. Accessed March 12, 2016.

37. White WB, Zannad F. Saxagliptin, alogliptin, and cardiovascular outcomes. N Engl J Med. 2014;370(5):484.

38. Gokhale M, Buse JB, Gray CL, Pate V, Marquis MA, Stürmer T. Dipeptidyl-peptidase-4 inhibitors and pancreatic cancer: a cohort study. Diabetes Obes Metab. 2014;16(12):1247-1256.

39. Gokhale M, Buse J, Sturmer T. Effect of body mass index on choice of initiating diabetes therapies. Pharmacoepidemiology and Drug Safety. Special Issue: Abstracts of the 30th International Conference on Pharmacoepidemiology and Therapeutic Risk Management, October 24-27, 2014, Taipei, Taiwan. ;23.

\section{SUPPORTING INFORMATION}

Additional supporting information may be found online in the Supporting Information section at the end of the article. 